\title{
Flipping bridge surfaces and bounds on the stable bridge number
}

\author{
JESSE JOHNSON \\ MAGGY TOMOVA
}

\begin{abstract}
We show that if $K$ is a knot in $S^{3}$ and $\Sigma$ is a bridge sphere for $K$ with high distance and $2 n$ punctures, the number of perturbations of $K$ required to interchange the two balls bounded by $\Sigma$ via an isotopy is $n$. We also construct a knot with two different bridge spheres with $2 n$ and $2 n-1$ bridges respectively for which any common perturbation has at least $3 n-4$ bridges. We generalize both of these results to bridge surfaces for knots in any 3 -manifold.
\end{abstract}

57M25, 57M27, 57M50

\section{Introduction}

Reidemeister [6] and Singer [8] showed that any two Heegaard splittings for a 3manifold $M$ have a common stabilization, ie, if $\Sigma$ and $\Sigma^{\prime}$ are two Heegaard surfaces for $M$ there exists a Heegaard surface $\Sigma^{\prime \prime}$ that is isotopic to a stabilization of $\Sigma$ as well as to a stabilization of $\Sigma^{\prime}$. A long standing question in Heegaard splittings asks what is the minimal genus of $\Sigma^{\prime \prime}$ in terms of the genera of $\Sigma$ and $\Sigma^{\prime}$. Examples of Heegaard splittings that required many stabilizations were presented by Bachman [1], Hass, Thompson and Thurston [3] and Johnson [5].

Bridge splittings are the natural extension of Heegaard splittings in the context of a compact orientable manifold $M$ containing a properly embedded tangle $T$. A bridge splitting for $(M, T)$ is a triple $\left(\Sigma,\left(H^{+}, \tau^{+}\right),\left(H^{-}, \tau^{-}\right)\right)$where $\Sigma$ is a connected surface that decomposes $M$ into compression bodies $H^{+}$and $H^{-}$and decomposes $T$ into collections of arcs $\tau^{+}$and $\tau^{-}$that are embedded in the corresponding compression bodies in specific ways. The surface $\Sigma$ is called a bridge surface for $(M, T)$. Note that if $T=\varnothing$, then $\left(\Sigma,\left(H^{+}, \tau^{+}\right),\left(H^{-}, \tau^{-}\right)\right)$is a Heegaard splitting for $M$.

Given a bridge surface $\Sigma$ of $(M, T)$ one can always obtain another bridge surface $\Sigma^{\prime \prime}$ by performing stabilizations and perturbations. These operations are discussed in detail by Scharlemann and Tomova [7] and they behave in a manner similar to stabilizations of Heegaard splittings. In this paper we consider pairs of bridge splittings $\Sigma$ and $\Sigma^{\prime}$ 
for $(M, T)$ and study bridge splittings $\Sigma^{\prime \prime}$ that can be obtained from both $\Sigma$ and $\Sigma^{\prime}$ via stabilizations and perturbations. The results we obtain are similar but somewhat weaker than the results obtained by Johnson for Heegaard splittings in [5] due to the additional difficulties introduced by the presence of the knot.

At first we will distinguish a bridge splitting $\left(\Sigma,\left(H^{+}, \tau^{+}\right),\left(H^{-}, \tau^{-}\right)\right)$from the bridge splitting $\left(\Sigma,\left(H^{-}, \tau^{-}\right),\left(H^{+}, \tau^{+}\right)\right)$in which the order of the compression bodies is reversed. We ask what is the minimum value of $2-\chi\left(\Sigma^{\prime \prime}\right)$ such that the bridge splitting $\left(\Sigma^{\prime \prime},\left(H^{\prime \prime+}, \tau^{\prime \prime+}\right),\left(H^{\prime \prime-}, \tau^{\prime \prime-}\right)\right)$ is isotopic to stabilizations and perturbations of both bridge splittings $\left(\Sigma,\left(H^{+}, \tau^{+}\right),\left(H^{-}, \tau^{-}\right)\right)$and $\left(\Sigma,\left(H^{-}, \tau^{-}\right),\left(H^{+}, \tau^{+}\right)\right)$. This value is called the flip Euler characteristic of $\Sigma$ and it is analogous to the flip genus of a Heegaard splitting defined in [5]. We give a bound on this quantity in terms of the Euler characteristic of $\Sigma$ and the distance of $T$ with respect to $\Sigma$ (Definition 4.1).

Theorem 1.1 Let $T$ be a prime tangle properly embedded in a compact orientable irreducible 3-manifold $M$ and let $\left(\Sigma,\left(H^{+}, \tau^{+}\right),\left(H^{-}, \tau^{-}\right)\right)$be a bridge splitting for $(M, T)$ such that $\chi(\Sigma) \leq-4$. Then the flip Euler characteristic of $\Sigma$ is at least $\max \{2-2 \chi(\Sigma), d(\Sigma, T)\}$.

Corollary 1.2 Let $T$ be a prime knot in $S^{3}$ and let $\Sigma$ be a bridge sphere for $T$ with $n \geq 3$ bridges such that $d(T, \Sigma) \geq 4 n$. If $\left(\Sigma^{\prime \prime},\left(H^{\prime \prime+}, \tau^{\prime \prime+}\right),\left(H^{\prime \prime-}, \tau^{\prime \prime-}\right)\right)$ is a minimal bridge number perturbation of both bridge splittings $\left(\Sigma,\left(H^{+}, \tau^{+}\right),\left(H^{-}, \tau^{-}\right)\right)$and $\left(\Sigma,\left(H^{-}, \tau^{-}\right),\left(H^{+}, \tau^{+}\right)\right)$, then $T$ has exactly $2 n-1$ bridges with respect to $\Sigma^{\prime \prime}$.

We next consider the problem of distinguishing bridge surfaces without keeping track of the order of the compression bodies. To make this clear, we will consider only the bridge surface rather than the bridge splitting. In this case we obtain the following result.

Theorem 1.3 There exist infinitely many manifolds $M_{\alpha}$ each containing a knot $K_{\alpha}$ so that each pair $\left(M_{\alpha}, K_{\alpha}\right)$ has two bridge surfaces $\Sigma$ and $\Sigma^{\prime}$ with $\chi(\Sigma)=2 s$ and $\chi\left(\Sigma^{\prime}\right)=2 s-2$ so that if $\Sigma^{\prime \prime}$ is bridge surface that is isotopic to stabilizations and perturbations of both $\Sigma$ and $\Sigma^{\prime}$, then $\chi\left(\Sigma^{\prime \prime}\right) \leq 3 s+4$.

As a corollary of the above we obtained the following result:

Corollary 1.4 For every $n \geq 2$ there exists a knot $\tilde{K}$ in $S^{3}$ with bridge spheres $\Sigma$ and $\Sigma^{\prime}$ with bridge numbers $2 n-1$ and $2 n$ respectively such that every bridge surface $\Sigma^{\prime \prime}$ that is isotopic to a perturbation of both has at least $3 n-4$ bridges. 
In Section 2 we give the definition of a bridge splitting for a pair $(M, T)$ and explain how a sweepout is associated to any bridge splitting. Furthermore we define two conditions on a pair of sweepouts: A sweepout $g$ can split a second sweepout $f$ for the same manifold or can span it. Generically these are the only two options for how $g$ behaves with respect to $f$.

In Section 3 and Section 4 we consider two bridge splittings $\Sigma$ and $\Sigma^{\prime}$ for $(M, T)$ with associated sweepouts $f$ and $g$. We show that if $g$ spans $f$, then the Euler characteristic of the punctured bridge surface $\Sigma$ is bounded below by the Euler characteristic of the punctured bridge surface $\Sigma^{\prime}$. Next we define the distance of a bridge splitting and we show that if $g$ splits $f$ then the distance of $\Sigma$ is bounded above by the Euler characteristic of $\Sigma^{\prime}$. Finally we consider the case where $g$ neither spans not splits $f$ and we show that this can only occur if $\chi(\Sigma) \geq-3$. Using these results, in Section 5 we prove Theorem 1.1 and in Section 6 we prove Theorem 1.3.

\section{Preliminaries}

\subsection{Compression bodies containing trivial arcs}

Let $H$ be a compression body. Recall that a spine of $H$ is a complex $\partial_{-} H \cup \Gamma$ where $\Gamma \subset H$ is a properly embedded finite graph with no valence 1 vertices in the interior of $H$ and such that $H$ is isotopic to a regular neighborhood of $\partial_{-} H \cup \Gamma$. A set of properly embedded $\operatorname{arcs} \tau=\left\{t_{1}, \ldots, t_{n}\right\}$ in $H$ is trivial if each $t_{i}$ is either parallel to $\partial_{+} H$ or is a vertical arc with one endpoint in $\partial_{+} H$ and the other endpoint in $\partial_{-} H$. If an arc is parallel to $\partial_{+} H$ the disk of parallelism is called a bridge disk. We will denote the pair of a compression body $H$ containing properly embedded trivial arcs $\tau$ by $(H, \tau)$. The $\operatorname{arcs} \tau$ can be isotoped in $H$ so that the projection $H-\operatorname{spine}(H) \cong \partial H \times[0,1) \rightarrow[0,1)$ has no critical points in the vertical arcs and a single critical point, say a maximum, in each boundary parallel arc. Let $s_{i}$ be a collection of vertical arcs each connecting a single maximum of $\tau$ to a spine of $H$. Let spine $((H, \tau))=\operatorname{spine}(H) \cup\left\{s_{i}\right\}$ and note that there is a map $(\partial H, \partial H \cap \tau) \times I \rightarrow(H, \tau)$ which is a homeomorphism except over the spine, and the map gives a neighborhood of the spine a mapping cylinder structure.

\subsection{Bridge splittings}

Let $T$ be a properly embedded tangle in a compact oriented 3-manifold $M$ and let $\Sigma$ be a properly embedded surface transverse to $T$ such that $\Sigma$ splits $M$ into two compression bodies $H^{+}$and $H^{-}$and such that $\tau^{+}=H^{+} \cap T$ and $\tau^{-}=H^{-} \cap T$ 
are trivial arcs in the corresponding compression body. In this case we say that $\left(\Sigma,\left(H^{+}, \tau^{+}\right),\left(H^{-}, \tau^{-}\right)\right)$is a bridge splitting for $(M, T)$ and $\Sigma$ is a bridge surface. As every compact orientable 3-manifold has a Heegaard splitting, it is easy to see that every properly embedded tangle in any 3 -manifold has a bridge splitting.

\subsection{Surfaces in $(M, T)$}

Suppose $M$ is a compact, irreducible, orientable 3-manifold containing a properly embedded tangle $T$ and let $F$ be a surface in $M$ transverse to $T$. The surface $F$ gives rise to a punctured surface in the complement of a regular neighborhood $\eta(T)$ of $T$. We will refer to this punctured surface as $F$ also and we will specify if we are referring to the punctured or the closed surface whenever it is not clear from context. Two surfaces in $(M, T)$ will be considered isotopic only if there is an isotopy between them transverse to the tangle.

A simple closed curve in $F-\eta(T)$ is essential if it does not bound a disk in $F$ and it is not parallel to the boundary of a puncture. A properly embedded arc in $F$ with endpoints in $F \cap \partial M$ is essential if it does not cobound a disk with an arc in $F \cap \partial M$. An embedded disk $D$ in $M$ is a compressing disk for $F$ if $D \cap T=\varnothing, D \cap F=\partial D$ and $\partial D$ is an essential curve in $F-\eta(T)$. A properly embedded disk $D^{c}$ in $M$ is a cut-disk for $F$ if $D^{c} \cap T$ is a single point in the interior of $D^{c}, D^{c} \cap F=\partial D^{c}$ and $\partial D^{c}$ is an essential curve in $F-\eta(T)$. A $c$-disk is either a cut or a compressing disk.

\subsection{Obtaining new bridge splittings from known ones}

We will consider two geometric operations which allow us to produce new bridge surfaces from existing ones. These are generalizations of stabilizations for Heegaard splittings. Following Hayashi and Shimokawa [4], the bridge surface $\Sigma$ will be called stabilized if there is a pair of compressing disks on opposite sides of $\Sigma$ that intersect in a single point. The bridge surface is called perturbed if there is a pair of bridge disks $D_{i}$ on opposite sides of $\Sigma$ such that $\varnothing \neq\left(\partial D_{1} \cap \partial D_{2}\right) \subset(\Sigma \cap T)$ and $\left|\partial D_{1} \cap \partial D_{2}\right|=1$. These operations are discussed in detail in [7].

\subsection{Sweepouts}

Suppose $(M, T)=\left(H^{+}, \tau^{+}\right) \cup_{\Sigma}\left(H^{-}, \tau^{-}\right)$. From the definition of a spine one can construct a map $f: M \rightarrow[-1,1]$ such that $f^{-1}(1)$ is isotopic to a spine of $\left(H^{+}, \tau^{+}\right)$, $f^{-1}(-1)$ is isotopic to a spine of $\left(H^{-}, \tau^{-}\right)$and $f^{-1}(t)$ is a surface isotopic to the punctured surface $\Sigma$ for every $t \in(-1,1)$. This function is called a sweepout representing $\left(\Sigma,\left(H^{-}, \tau^{-}\right),\left(H^{+}, \tau^{+}\right)\right)$. 
We give a brief overview of how sweepouts can be applied to study bridge surfaces for tangles in a 3-manifold. Further details can be found in [10]. Consider a tangle properly embedded in a 3-manifold with two bridge splittings. Let $f$ be a sweepout representing the bridge splitting $\left(\Sigma,\left(H^{-}, \tau^{-}\right),\left(H^{+}, \tau^{+}\right)\right)$and let $g$ be another sweepout representing a second bridge splitting for $(M, T)$ which we denote $\left(\Sigma^{\prime},\left(H^{\prime-}, \tau^{\prime-}\right),\left(H^{\prime+}, \tau^{\prime+}\right)\right)$.

Consider the two parameter sweepout $f \times g$ that maps $(M, T)$ into the square $[-1,1] \times[-1,1]$. Each point $(s, t)$ in the square represents a pair of surfaces: $\Sigma_{t}=$ $f^{-1}(t)-\eta(T)$ isotopic to the punctured surface $\Sigma$ and $\Sigma_{s}^{\prime}=g^{-1}(s)-\eta(T)$ isotopic to $\Sigma^{\prime}$. The graphic is the subset $\Gamma$ of the square consisting of all points $(s, t)$ where either $\Sigma_{t}$ is tangent to $\Sigma_{s}^{\prime}$ or $\Sigma_{t} \cap \Sigma_{s}^{\prime}$ contains a point of $T$. We say that $f \times g$ is generic if it is stable on the complement of the spines and each arc $\{t\} \times[-1,1]$ and $[-1,1] \times\{s\}$ contains at most one vertex of the graphic. If $f \times g$ is generic then at each (valence four) vertex of $\Gamma$ there are two points of tangency, two points of $T$ in the intersection, or one of each. By general position of the spines $f^{-1}( \pm 1)$ with the surface $\Sigma^{\prime}$, the graphic $\Gamma$ is incident to $\partial I \times I$ in only a finite number of points corresponding to tangencies between $f^{-1}( \pm 1)$ and $\Sigma^{\prime}$.

\subsection{Splitting and spanning sweepouts}

Suppose $f$ and $g$ are sweepouts for $(M, T)$ and $f \times g$ is generic. Generalizing [5], for some fixed values of $s$ and $t$ we will say that $\Sigma_{t}$ is mostly above $\Sigma_{s}^{\prime}$ if each component of $\Sigma_{t} \cap H_{s}^{\prime-}$ (if there are any) is contained in a disk or a once-punctured disk in $\Sigma_{t}$. Similarly, we will say that $\Sigma_{t}$ is mostly below $\Sigma_{s}^{\prime}$ if each component of $\Sigma_{t} \cap H_{s}^{\prime+}$ is contained in a disk or once-punctured disk in $\Sigma_{t}$. We will say that $g$ spans $f$ if there are values $t_{+}, t_{-}$and $s$ for which $\Sigma_{t_{+}}$is mostly above $\Sigma_{s}^{\prime}$ and $\Sigma_{t_{-}}$ is mostly below $\Sigma_{s}^{\prime}$. We will say that $g$ spans $f$ positively if $t_{-}<t_{+}$and negatively otherwise. These conditions are shown at the top of Figure 1. Note that $g$ may span $f$ both positively and negatively.

We will say that $g$ splits $f$ if there is a value of $s$ such that the horizontal line $[-1,1] \times\{s\} \subset[-1,1] \times[-1,1]$ does not intersect any vertices of $\Gamma$ and for every $t$ the surface $\Sigma_{t}$ is neither mostly above nor mostly below $\Sigma_{s}^{\prime}$. This is shown at the bottom left of Figure 1. Note that this condition is equivalent to the condition that there exists an $s$ such that for every $t, \Sigma_{s}^{\prime} \cap \Sigma_{t}$ contains at least one curve that is essential in $\Sigma_{t}$.

\section{Spanning sweepouts and bounds on Euler characteristic}

As in the last section, we will let $f$ and $g$ be sweepouts for the pair $(M, T)$ associated to the two bridge splittings $\left(\Sigma,\left(H^{-}, \tau^{-}\right),\left(H^{+}, \tau^{+}\right)\right)$and $\left(\Sigma^{\prime},\left(H^{\prime-}, \tau^{\prime-}\right),\left(H^{\prime+}, \tau^{\prime+}\right)\right)$ 

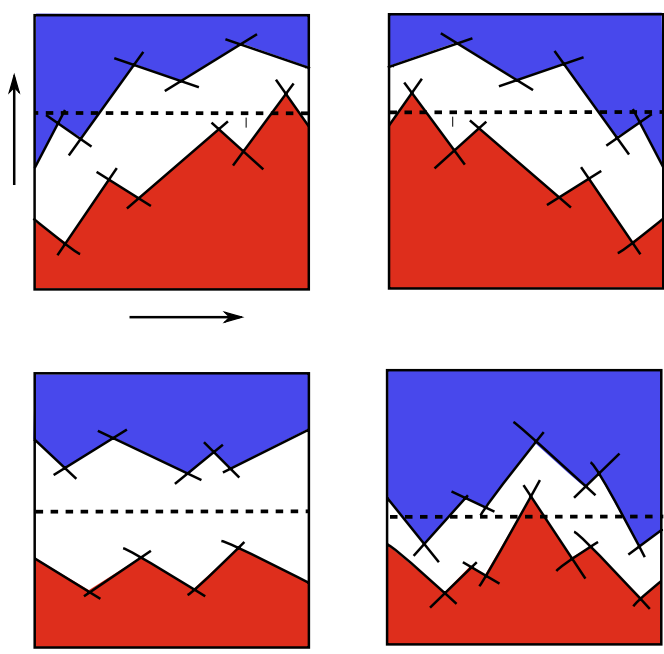

Figure 1: The blue regions are those where $\Sigma_{t}$ is mostly below $\Sigma_{s}^{\prime}$ and the red regions are where $\Sigma_{t}$ is mostly above $\Sigma_{s}^{\prime}$. The figures show $g$ spanning $f$ positively, negatively, both positively and negatively and $g$ splitting $f$.

respectively. Define $\Sigma_{t}=f^{-1}(t)-\eta(T)$, and $\Sigma_{s}^{\prime}=g^{-1}(s)-\eta(T)$ for $s, t \in(-1,1)$. We will also name the compression bodies $H_{s}^{\prime-}=g^{-1}(-1, s]$ containing the trivial $\operatorname{arcs} \tau_{s}^{\prime-}$ and $H_{s}^{\prime+}=g^{-1}[s, 1)$ containing the trivial arcs $\tau_{s}^{\prime+}$.

Theorem 3.1 Let $f$ and $g$ be sweepouts associated to bridge surfaces $\Sigma$ and $\Sigma^{\prime}$ for a prime, unsplit tangle $T$ in an irreducible orientable 3-manifold $M$. Suppose that $f \times g$ is generic and suppose that there are values $s$ and $t_{+}>t_{0}$ such that $\Sigma_{t_{+}}$is mostly above $\Sigma_{s}^{\prime}$ and $\Sigma_{t_{0}}$ is mostly below $\Sigma_{s}^{\prime}$. Then there is a sequence of compressions and cut compressions of $\Sigma_{s}^{\prime}$ after which there is a component of the compressed surface which is parallel to $\Sigma$. The parallelism is supported in the complement of the spines of $\Sigma$.

If there are values $s$ and $t_{+}>t_{0}>t_{-}$such that $\Sigma_{t_{+}}$and $\Sigma_{t_{-}}$are mostly above $\Sigma_{s}^{\prime}$ and $\Sigma_{t_{0}}$ is mostly below $\Sigma_{s}^{\prime}$ then there is a sequence of compressions and cut compressions of $\Sigma^{\prime}$ after which there are two components of the compressed surface which are parallel to $\Sigma$. The parallelism is supported in the complement of the spines of $\Sigma$.

Because the Euler characteristic is nondecreasing under c-compression, this theorem implies the following corollary: 
Corollary 3.2 Let $f$ and $g$ be sweepouts associated to bridge surfaces $\Sigma$ and $\Sigma^{\prime}$ for a prime, unsplit tangle $T$ in an irreducible orientable 3-manifold $M$. If $f \times g$ is generic and $g$ spans $f$ positively (or negatively) then $\chi\left(\Sigma^{\prime}\right) \leq \chi(\Sigma)$. If $g$ spans $f$ both positively and negatively then $\chi\left(\Sigma^{\prime}\right) \leq 2 \chi(\Sigma)$.

Proof of Theorem 3.1 We will only prove the second statement as the proof of the first statement is similar but simpler. Suppose there are values $s$ and $t_{+}>t_{0}>t_{-}$such that $\Sigma_{t_{+}}$and $\Sigma_{t_{-}}$are mostly above $\Sigma_{s}^{\prime}$ and $\Sigma_{t_{0}}$ is mostly below $\Sigma_{s}^{\prime}$.

By definition, each loop in the intersection $\Sigma_{s}^{\prime} \cap\left(\Sigma_{t_{+}} \cup \Sigma_{t_{0}} \cup \Sigma_{t_{-}}\right)$bounds a disk or a once-punctured disk in $\Sigma_{t_{+}} \cup \Sigma_{t_{0}} \cup \Sigma_{t_{-}}$. To facilitate this discussion color $H_{s}^{\prime-}$ blue and $H_{s}^{\prime+}$ red. This induces a coloring on $\Sigma_{t_{+}}, \Sigma_{t_{-}}$and $\Sigma_{t_{0}}$. As $\Sigma_{t_{+}}$and $\Sigma_{t_{-}}$ are mostly above $\Sigma_{s}^{\prime}$ every component of $\left(\Sigma_{t_{+}} \cup \Sigma_{t_{-}}\right)-\Sigma_{s}^{\prime}$ that is not contained in a possibly once punctured subdisk of $\Sigma_{t_{+}}$or $\Sigma_{t_{-}}$is red. Every component of $\Sigma_{t_{0}}-\Sigma_{s}^{\prime}$ that is not contained in a possibly once punctured subdisk of $\Sigma_{t_{0}}$ is blue.

Let $\ell$ be a curve of $\Sigma_{s}^{\prime} \cap \Sigma_{t_{+}}$that is innermost in $\Sigma_{t_{+}}$. By hypothesis, $\ell$ is necessarily inessential in $\Sigma_{t_{+}}$. If $\ell$ is also inessential in $\Sigma_{s}^{\prime}$ then it can be removed by an isotopy of $\Sigma_{s}^{\prime}$ as $M$ is irreducible and $T$ is prime. If $\ell$ is essential in $\Sigma_{s}^{\prime}$ then the possibly punctured disk it bounds in $\Sigma_{t_{+}}$is a c-disk for $\Sigma_{s}^{\prime}$. Replace $\Sigma_{s}^{\prime}$ with the surface $F_{0}$ that results from c-compressing $\Sigma_{s}^{\prime}$ along this c-disk. Note that neither of these two moves affects the coloring of any region of $\Sigma_{t_{+}}-\Sigma_{s}^{\prime}$ that is not contained in a possibly punctured subdisk of $\Sigma_{t_{+}}$. We can then repeat this construction with an innermost loop of $F_{0} \cap \Sigma_{t_{+}}$, producing a surface $F_{1}$ and so on until we find a surface $F_{k}$ disjoint from $\Sigma_{t_{+}}$. At the end of this sequence of isotopies and c-compressions, $\Sigma_{t_{+}}$will be entirely red.

Repeat the above process with $\Sigma_{t_{-}}$and $F_{k}$ playing the roles of $\Sigma_{t_{+}}$and $\Sigma_{s}^{\prime}$ respectively to obtain a surface $F_{\ell}$ disjoint from both $\Sigma_{t_{+}}$and $\Sigma_{t_{-}}$and leaving $\Sigma_{t_{-}}$entirely red. Finally repeat the process beginning with $\Sigma_{t_{0}}$ and $F_{\ell}$ to obtain a surface $F_{m}$ disjoint from all of $\Sigma_{t_{+}} \cup \Sigma_{t_{0}} \cup \Sigma_{t_{-}}$and leaving $\Sigma_{t_{0}}$ entirely blue.

Maximally c-compress the surface $F_{n}=F_{m} \cap f^{-1}\left(t_{-}, t_{+}\right)$in the complement of $\Sigma_{t_{+}} \cup \Sigma_{t_{0}} \cup \Sigma_{t_{-}}$to get a surface $\tilde{F}$. Each component of $\tilde{F}$ is contained in a 3manifold homeomorphic to $\Sigma \times I$ and is c-incompressible in this manifold. By [11, Corollary 3.7] each component of $\widetilde{F}$ is either a sphere disjoint from $T$, a sphere bounding a ball containing a trivial subarc of $T$ or a component parallel to $\Sigma_{t_{0}}$. Note that $\tilde{F}$ was obtained from $\Sigma_{s}^{\prime}$ by c-compressions and therefore it cannot have sphere components disjoint from $T$ as $\Sigma_{s}^{\prime}$ does not have any such component, ie, all components of $\tilde{F}$ have nonpositive Euler characteristic. In addition $\widetilde{F}$ separates $\Sigma_{t_{0}}$ from $\Sigma_{t_{+}}$and $\Sigma_{t_{0}}$ from $\Sigma_{t_{-}}$as $\Sigma_{t_{0}}$ is entirely blue and $\Sigma_{t_{+}}$and $\Sigma_{t_{-}}$are red. 
Therefore $\widetilde{F}$ must have at least two components parallel to $\Sigma_{t_{0}}$, one lying in the product region between $\Sigma_{t_{-}}$and $\Sigma_{t_{0}}$ and one lying in the product region between $\Sigma_{t_{0}}$ and $\Sigma_{t_{+}}$.

\section{Splitting sweepouts and bounds on distance}

We briefly review the definition of distance of a bridge surface. For more details, see Tomova [10].

Definition 4.1 Suppose $M$ is a compact, orientable, irreducible 3-manifold containing a properly embedded tangle $T$ and suppose $\left(\Sigma,\left(H^{-}, \tau^{-}\right),\left(H^{+}, \tau^{+}\right)\right)$is a bridge splitting for $(M, T)$. The curve complex $\mathcal{C}(\Sigma, T)$ is a graph with vertices corresponding to isotopy classes of essential simple closed curves in $\Sigma-\eta(T)$. Two vertices are adjacent in $\mathcal{C}(\Sigma, T)$ if their corresponding classes of curves have disjoint representatives.

Let $\mathcal{V}^{+}\left(\operatorname{resp} \mathcal{V}^{-}\right)$be the set of all essential simple closed curves in $\Sigma-\eta(T)$ that bound disks in $H^{+}-\eta(T)$ (resp $H^{-}-\eta(T)$ ). Then the distance of the bridge splitting, $d(\Sigma, T)$, is the minimum distance between a vertex in $\mathcal{V}^{+}$and a vertex in $\mathcal{V}^{-}$measured in $\mathcal{C}(\Sigma, T)$ with the path metric.

Theorem 4.2 Let $f$ and $g$ be sweepouts associated to bridge surfaces $\Sigma$ and $\Sigma^{\prime}$ for a prime tangle $T$ in an irreducible 3-manifold $M$ and suppose that $\chi(\Sigma) \leq-1$. If $f \times g$ is generic and $g$ splits $f$ then $d(\Sigma, T) \leq 2-\chi\left(\Sigma^{\prime}\right)$.

Proof Let $s$ be such that for every $t \in(-1,1)$ the intersection $\Sigma_{s}^{\prime} \cap \Sigma_{t}$ contains a curve that is essential in $\Sigma_{t}$ and $[-1,1] \times\{s\}$ is disjoint from all vertices of $\Gamma$ so in particular $\left.g\right|_{\Sigma_{t}}$ is Morse. Let $H_{t}^{-}=f^{-1}[-1, t)$ and $H_{t}^{+}=f^{-1}(t, 1]$ be the two components of $M-\Sigma_{t}$. For values of $t$ close to -1 , all curves of $\Sigma_{s}^{\prime} \cap \Sigma_{t}$ bound disks in $H_{t}^{-}$because $\Sigma_{s}^{\prime}$ is transverse to the spine $f^{-1}(-1)$. Similarly for values of $t$ close to 1 , all curves of $\Sigma_{s}^{\prime} \cap \Sigma_{t}$ bound disks in $H_{t}^{+}$.

If for some value $t$ there is a curve of $\Sigma^{\prime} \cap \Sigma_{t}$ that is essential in $\Sigma_{t}$ and bounds a disk in $H_{t}^{+}$and simultaneously there is a curve of $\Sigma^{\prime} \cap \Sigma_{t}$ that is essential in $\Sigma_{t}$ and bounds a disk in $H_{t}^{-}$, then $d(\Sigma, T) \leq 1$.

We will say that two values $t_{-}, t_{+}$are adjacent if there is a single critical value for $f$ between them. In this case, the projections of the curves $\Sigma^{\prime} \cap \Sigma_{t_{-}}$to $\Sigma_{t_{+}}$can be isotoped disjoint from the curves $\Sigma^{\prime} \cap \Sigma_{t_{+}}$. Thus, if for some adjacent values $t_{-}, t_{+}$, there is a curve of $\Sigma^{\prime} \cap \Sigma_{t_{-}}$that is essential in $\Sigma_{t_{-}}$and bounds a disk in $H_{t_{-}}^{-}$and a curve of $\Sigma^{\prime} \cap \Sigma_{t_{+}}$that is essential in $\Sigma_{t_{+}}$and bounds a disk in $H_{t_{+}}^{+}$, we can again conclude that $d(\Sigma, T) \leq 1$. 
The above discussion shows that either $d(\Sigma, T) \leq 1 \leq 2-\chi\left(\Sigma^{\prime}\right)$ or there is an interval $[\alpha, \beta]$, where $\alpha \neq \beta$ are critical values for $\left.f\right|_{\Sigma_{s}^{\prime}}$ such that for every $t \in(\alpha, \beta)$, no curve of $\Sigma_{s}^{\prime} \cap \Sigma_{t}$ is both essential in $\Sigma_{t}$ and bounds a disk in $\Sigma_{s}^{\prime}$. Moreover for a very small $\epsilon, \Sigma_{S}^{\prime} \cap \Sigma_{\alpha-\epsilon}$ contains a curve that is essential in $\Sigma_{\alpha-\epsilon}$ and bounds a disk in $H_{\alpha-\epsilon}^{-}$and $\Sigma_{S}^{\prime} \cap \Sigma_{\beta+\epsilon}$ contains a curve that is essential in $\Sigma_{\beta+\epsilon}$ and bounds a disk in $H_{\beta+\epsilon}^{+}$.

Let $\alpha^{\prime}$ be just above $\alpha$ and $\beta^{\prime}$ be just below $\beta$. Suppose some component of $\Sigma_{s}^{\prime} \cap \Sigma_{\alpha^{\prime}}$ bounds a disk in $\Sigma_{s}^{\prime}$. Then this component must also bound a disk in $\Sigma_{\alpha^{\prime}}$ and therefore $\Sigma_{s}^{\prime}$ can be isotoped to remove this component. After some number of isotopies we obtains a surface $\Sigma^{\prime \prime}$ so that no curve of $\Sigma^{\prime \prime} \cap \Sigma_{\alpha^{\prime}}$ or $\Sigma^{\prime \prime} \cap \Sigma_{\beta^{\prime}}$ bounds a disk in $\Sigma^{\prime \prime}$. Define $S=\Sigma^{\prime \prime} \cap f^{-1}\left[\alpha^{\prime}, \beta^{\prime}\right]$. Because the boundary curves of $S$ do not bound disks in $\Sigma^{\prime \prime}$, it follows that $\chi(S) \geq \chi\left(\Sigma_{S}^{\prime}\right)$. Let $\pi$ be the projection map from $f^{-1}\left[\alpha^{\prime}, \beta^{\prime}\right]$ to $\Sigma_{0}$. By [5, Lemma 30], isotopy classes of loops in $S$ project to isotopy classes in $\Sigma_{0}$. Although we are now dealing with punctured surfaces the proof of this result is the same so we will not repeat it here.

As in [5] we let $L$ be the set of isotopy classes of loops of $\left.f\right|_{S}$ and let $\pi^{*}$ be the natural map from $L$ to $\mathcal{C}\left(\Sigma_{0}, T\right)$, together with $\{0\}$ where each curve in $L$ maps to the vertex that corresponds to its projection in $\Sigma_{0}$ unless it is inessential in $\Sigma_{0}$ in which case it is mapped to $\{0\}$.

Note that $L$ determines a decomposition of $S$ into pairs of pants and punctured annuli. Lemma 31 in [5] shows that if $\ell$ and $\ell^{\prime}$ are cuffs of the same pair of pants, then their images under $\pi^{*}$ are adjacent vertices in $\mathcal{C}\left(\Sigma_{0}, T\right)$. The same is true if $\ell$ and $\ell^{\prime}$ are the two boundary components of a punctured annulus. For if that is the case, then $\left.f\right|_{\Sigma^{\prime}}$ passes through a puncture so it contains a level component which is an arc with both of its endpoints lying in a boundary component of $\Sigma^{\prime}$. The projection of this component to $\Sigma_{0}$ is also an arc with both endpoints on some boundary component. The boundary curves of a regular neighborhood of the arc together with the boundary component are isotopic to the projections of $\ell$ and $\ell^{\prime}$ and thus $\ell$ and $\ell^{\prime}$ are disjoint.

Let $L^{\prime}=\pi_{*}(L) \cap \mathcal{C}\left(\Sigma_{0}, T\right)$. By [5, Lemma 32] this set is connected and has diameter equal to at most the number of components of $S-L$. Each component of $S-L$ is a punctured annulus or a pair of pants and therefore contributes -1 to $\chi(S)$. It follows that $\operatorname{diam}\left(L^{\prime}\right) \leq-\chi(S)$.

Recall that for a very small $\epsilon, \Sigma^{\prime} \cap \Sigma_{\alpha-\epsilon}$ contains a curve that bounds a compressing disk for $H_{\alpha-\epsilon}^{-}$and $\Sigma^{\prime} \cap \Sigma_{\beta+\epsilon}$ contains a curve that bounds a compressing disk for $H_{\beta+\epsilon}^{+}$. As the intervals $\left(\alpha-\epsilon, \alpha^{\prime}\right)$ and $\left(\beta^{\prime}, \beta+\epsilon\right)$ contain exactly one critical point each, every curve in the set $\pi\left(\Sigma^{\prime} \cap \Sigma_{\alpha-\epsilon}\right)$ is distance at most one from every 
curve in the set $\pi\left(\Sigma^{\prime} \cap \Sigma_{\alpha^{\prime}}\right)$ and similarly every curve in the set $\pi\left(\Sigma^{\prime} \cap \Sigma_{\beta+\epsilon}\right)$ is distance at most one from every curve in the set $\pi\left(\Sigma^{\prime} \cap \Sigma_{\beta^{\prime}}\right)$. Adding these distances we obtain the inequality $d(\Sigma) \leq \operatorname{diam}\left(L^{\prime}\right)+2 \leq 2-\chi(S) \leq 2-\chi\left(\Sigma^{\prime}\right)$ as desired.

In this and in the previous section we saw that if $f$ and $g$ are two sweepouts associated to bridge surfaces $\Sigma$ and $\Sigma^{\prime}$ for the pair $(M, T)$ and $g$ spans $f$, then we can relate $\chi(\Sigma)$ and $\chi\left(\Sigma^{\prime}\right)$ and if $g$ splits $f$ then we can relate $d(\Sigma, T)$ and $\chi\left(\Sigma^{\prime}\right)$. It is clear that if $g$ and $f$ are sweepouts such that $f \times g$ is generic, then either $g$ spans $f, g$ splits $f$ or there are values of $s$ and $t$ such that for a small $\epsilon, \Sigma_{t}$ is mostly above $\Sigma_{s-\epsilon}^{\prime}$ and $\Sigma_{t}$ is mostly below $\Sigma_{s+\epsilon}^{\prime}$. We now consider a slight generalization of this last case.

Lemma 4.3 Suppose $f$ and $g$ are sweepouts for a tangle in a manifold such that $f \times g$ is generic except possibly for a single vertex of order 6 or two vertices of order 4 with the same $s$ coordinate. Suppose the graphic of $f \times g$ has a vertex at coordinates $(s, t)$ such that for a small $\epsilon, \Sigma_{t}$ is mostly above $\Sigma_{s-\epsilon}^{\prime}$ and $\Sigma_{t}$ is mostly below $\Sigma_{s+\epsilon}^{\prime}$. If this vertex has valence 4 , then $\chi(\Sigma) \geq-2$. If the vertex has valence 6 , then $\chi(\Sigma) \geq-3$.

Proof By the definition of $f \times g$ it follows that, $\left.g\right|_{\Sigma_{t^{\prime}}}$ is Morse where $t^{\prime}=t+\epsilon^{\prime}$ for a small $\epsilon^{\prime}$. Furthermore there are two critical values for $\left.g\right|_{\Sigma_{t^{\prime}}}, a<b$ with at most one other critical value between them (if the valence of $(s, t)$ is 6 ) such that if $a^{\prime}$ is a regular value directly below $a$ and $b^{\prime}$ is a regular value directly above $b$, then $\Sigma_{t^{\prime}}$ is mostly above $\Sigma_{a^{\prime}}^{\prime}$ and mostly below $\Sigma_{b^{\prime}}^{\prime}$.

Consider first $\Sigma_{t^{\prime}} \cap \Sigma_{a^{\prime}}^{\prime}$. By definition each component of $\Sigma_{t^{\prime}} \cap H_{a^{\prime}}^{\prime-}$ is contained in a possibly punctured disk subset of $\Sigma_{t^{\prime}}$. Let $\Lambda$ be the set of all curves of $\Sigma_{t^{\prime}} \cap \Sigma_{a^{\prime}}^{\prime}$ that are not contained in the interior of a disk or punctured disk component of $\Sigma_{t^{\prime}}-\Sigma_{a^{\prime}}^{\prime}$; see Figure 2. Then $\Sigma_{t^{\prime}}-\Lambda$ is a collection of components all but one of which are possibly punctured disks. Note that the Euler characteristics of each of these possibly punctured disk components is at least 0 .

Passing through each critical point between $a^{\prime}$ and $b^{\prime}$ is equivalent to adding a band between two components of $\Sigma_{t^{\prime}}-\Sigma_{a^{\prime}}^{\prime}$ or banding a component to itself. In either case the sum of the Euler characteristics of all components is decreased by one. As these bands correspond to a sweepout they all lie on the same side of $\Sigma^{\prime}$. As $\Sigma_{t^{\prime}}$ is mostly below $\Sigma_{b^{\prime}}^{\prime}$, it follows that after attaching at most three bands to a collection of at most once punctured disks, the result is a surface isotopic to $\Sigma_{t^{\prime}}$ with possibly some disks and once punctured disks missing, ie $\Sigma_{t^{\prime}} \cap \Sigma_{b^{\prime}}^{\prime}$ is also as in Figure 2 but now the subsurface which is not contained in a punctured disk is below $\Sigma_{b^{\prime}}^{\prime}$. As at most three bands were added, it follows that $\chi\left(\Sigma_{t^{\prime}}\right) \geq-3$. If the vertex $(s, t)$ has valence four, then only two bands need to be added so $\chi\left(\Sigma_{t^{\prime}}\right) \geq-2$. 


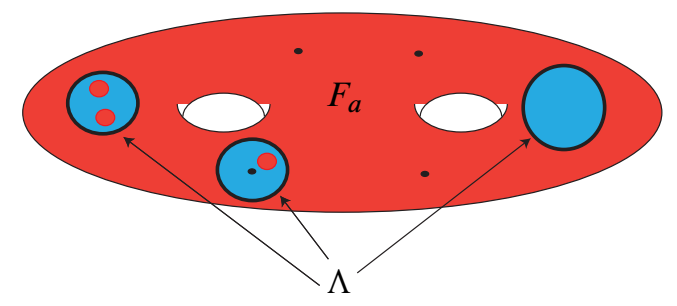

Figure 2

Using the results in this and the previous section we can obtain the following generalization of the main result in [10].

Theorem 4.4 Suppose $N$ is a manifold containing a tangle $K$ and let $M$ be a submanifold such that $T=K \cap M$ is a properly embedded tangle. Let $\Sigma$ be a bridge surface of $(M, T)$ and let $\Sigma^{\prime}$ be a bridge surface of $(N, K)$. Then one of the following holds:

- There is an isotopy of $\Sigma^{\prime}$ followed by some number of compressions and cutcompressions of $\Sigma^{\prime} \cap M$ in $M$ giving a compressed surface $\Sigma^{\prime \prime}$, such that at least one component of $\Sigma^{\prime \prime} \cap M$ is parallel to $\Sigma$, in the complement of the spines of $\Sigma$.

- $d(\Sigma, T) \leq 2-\chi\left(\Sigma^{\prime}\right)$.

- $\chi(\Sigma) \geq-2$.

Proof Because $(M, T) \subset(N, K)$ for values of $s$ close to $-1, \Sigma_{t}$ is mostly above $\Sigma_{s}^{\prime}$ and for values of $s$ close to $1, \Sigma_{t}$ is mostly below $\Sigma_{s}^{\prime}$. Therefore there are three possibilities. Either $g$ spans $f, g$ splits $f$ or the graphic of $f \times g$ has a vertex of valence 4 at coordinates $(s, t)$ such that for a small $\epsilon, \Sigma_{t}$ is mostly above $\Sigma_{s-\epsilon}^{\prime}$ and $\Sigma_{t}$ is mostly below $\Sigma_{s+\epsilon}^{\prime}$. Thus there are three cases to consider.

Case 1 If $g$ spans $f$ then there are values $s$ and $t_{+}$and $t_{-}$such that $\Sigma_{t_{+}}$is mostly above $\Sigma_{s}^{\prime}$ and $\Sigma_{t_{-}}$is mostly below $\Sigma_{s}^{\prime}$. By the arguments in Theorem 3.1 it follows that after some number of compressions and cut-compressions of $\Sigma_{s}^{\prime} \cap M$ in $M$ we obtain an incompressible surface $\Sigma^{\prime \prime}$ that separates $\Sigma_{t_{+}}$and $\Sigma_{t_{-}}$and therefore it is parallel to $\Sigma$ as desired.

Case 2 If $g$ splits $f$, then the arguments in the proof of Theorem 4.2 show that $d(\Sigma, T) \leq 2-\chi\left(\Sigma^{\prime}\right)$ as desired.

Case 3 Finally suppose that the graphic of $f \times g$ has a vertex at coordinates $(s, t)$ such that for a small $\epsilon, \Sigma_{t}$ is mostly above $\Sigma_{s-\epsilon}^{\prime}$ and $\Sigma_{t}$ is mostly below $\Sigma_{s+\epsilon}^{\prime}$. Then by Lemma 4.3 it follows that $\chi(\Sigma) \geq-2$. 


\section{Flipping bridge surfaces}

In this section we want to restrict our attention to oriented isotopies, ie, if $\Sigma$ and $\Sigma^{\prime}$ are bridge splittings for $(M, T)$ splitting the manifold into compression bodies $H^{+}, H^{-}$ and $H^{\prime+}, H^{-}$respectively, then the bridge splittings $\left(\Sigma,\left(H^{+}, \tau^{+}\right),\left(H^{-}, \tau^{-}\right)\right)$and $\left(\Sigma^{\prime},\left(H^{\prime+}, \tau^{\prime+}\right),\left(H^{\prime-}, \tau^{\prime-}\right)\right)$ will be called orientation isotopic if there is an isotopy mapping $\Sigma$ to $\Sigma^{\prime},\left(H^{+}, \tau^{+}\right)$to $\left(H^{\prime+}, \tau^{\prime+}\right)$ and $\left(H^{-}, \tau^{-}\right)$to $\left(H^{\prime-}, \tau^{\prime-}\right)$. Following [5] we will say that a bridge surface $\Sigma$ is flippable if $\left(\Sigma,\left(H^{+}, \tau^{+}\right),\left(H^{-}, \tau^{-}\right)\right)$is orientation isotopic to $\left(\Sigma,\left(H^{-}, \tau^{-}\right),\left(H^{+}, \tau^{+}\right)\right)$.

Suppose $\left(\Sigma^{\prime},\left(H^{\prime+}, \tau^{\prime+}\right),\left(H^{\prime-}, \tau^{\prime-}\right)\right)$ is a bridge splitting for $(M, T)$ isotopic to stabilizations and perturbations of both bridge splittings $\left(\Sigma,\left(H^{+}, \tau^{+}\right),\left(H^{-}, \tau^{-}\right)\right)$and $\left(\Sigma,\left(H^{-}, \tau^{-}\right),\left(H^{+}, \tau^{+}\right)\right)$. The minimal value of $2-\chi\left(\Sigma^{\prime}\right)$ is called the flip Euler characteristic of $\Sigma$ and it is analogous to the flip genus of a Heegaard splitting defined in [5].

We will take advantage of several results previously proven for sweepouts of Heegaard splittings. The proofs carry over with only minor modifications.

Lemma 5.1 (See $\left[5\right.$, Lemmas 14 and 15].) If $\left(\Sigma,\left(H^{+}, \tau^{+}\right),\left(H^{-}, \tau^{-}\right)\right)$is a bridge decomposition for some knot $K \subset M$ then $\left(\Sigma,\left(H^{+}, \tau^{+}\right),\left(H^{-}, \tau^{-}\right)\right)$spans itself positively. If $\left(\Sigma^{\prime},\left(H^{\prime+}, \tau^{\prime+}\right),\left(H^{\prime-}, \tau^{\prime-}\right)\right)$ is either a perturbation or stabilization of $\left(\Sigma,\left(H^{+}, \tau^{+}\right),\left(H^{-}, \tau^{-}\right)\right)$, then it spans the bridge splittings $\left(\Sigma,\left(H^{+}, \tau^{+}\right),\left(H^{-}, \tau^{-}\right)\right)$ positively and spans $\left(\Sigma,\left(H^{-}, \tau^{-}\right),\left(H^{+}, \tau^{+}\right)\right)$negatively.

Proof Let $f$ be a sweepout for the decomposition $\left(\Sigma,\left(H^{+}, \tau^{+}\right),\left(H^{-}, \tau^{-}\right)\right)$. Then there is a second sweepout $g$ for $\left(\Sigma,\left(H^{+}, \tau^{+}\right),\left(H^{-}, \tau^{-}\right)\right)$such that $g^{-1}(0)=\Sigma_{0}^{\prime}$ is disjoint from and separates the spines $f^{-1}(-1), f^{-1}(1)$. Thus for $t_{-}$near -1 and $t_{+}$near $1, \Sigma_{t_{-}}$will be mostly below $\Sigma_{0}^{\prime}$ and $\Sigma_{t_{+}}$will be mostly above. This implies that $\left(\Sigma,\left(H^{+}, \tau^{+}\right),\left(H^{-}, \tau^{-}\right)\right)$spans itself positively.

Similarly, for any perturbation or stabilization of the bridge decomposition, we can perturb or stabilize $\Sigma_{0}^{\prime}$ while keeping it disjoint from $f^{-1}(-1), f^{-1}(1)$, then extend this surface to a sweepout for the perturbed or stabilized bridge decomposition. Therefore if $\left(\Sigma^{\prime},\left(H^{\prime+}, \tau^{\prime+}\right),\left(H^{\prime-}, \tau^{\prime-}\right)\right)$ is a perturbation or stabilization of $\left(\Sigma,\left(H^{+}, \tau^{+}\right),\left(H^{-}, \tau^{-}\right)\right)$, then it spans $\left(\Sigma,\left(H^{+}, \tau^{+}\right),\left(H^{-}, \tau^{-}\right)\right)$positively and spans $\left(\Sigma,\left(H^{-}, \tau^{-}\right),\left(H^{+}, \tau^{+}\right)\right)$negatively.

Let $\Sigma^{\prime}$ be a common stabilization or perturbation of $\left(\Sigma,\left(H^{-}, \tau^{-}\right),\left(H^{+}, \tau^{+}\right)\right)$and $\left(\Sigma,\left(H^{+}, \tau^{+}\right),\left(H^{-}, \tau^{-}\right)\right)$. In particular there are sweepouts $g$ and $g^{\prime}$ representing $\left(\Sigma^{\prime},\left(H^{\prime-}, \tau^{\prime-}\right),\left(H^{\prime+}, \tau^{\prime+}\right)\right)$, and a sweep out $f$ representing $\left(\Sigma,\left(H^{-}, \tau^{-}\right),\left(H^{+}, \tau^{+}\right)\right)$ 
such that $g$ spans $f$ positively and $g^{\prime}$ spans $f$ negatively. As $g$ and $g^{\prime}$ represent the same bridge decomposition and are therefore orientation isotopic it follows that there is a family of sweepouts $\left\{g_{r} \mid r \in[0,1]\right\}$ such that $g_{0}=g, g_{1}=g^{\prime}$.

Lemma 5.2 [5, Lemma 34] Let $g$ and $g^{\prime}$ be sweepouts such that $f \times g$ and $f \times g^{\prime}$ are generic and $g$ is isotopic to $g^{\prime}$. There is a family of sweepouts $\left\{g_{r} \mid r \in[0,1]\right\}$ such that $g_{0}=g, g_{1}=g^{\prime}$ and for all but finitely many $r, f \times g_{r}$ is generic. At the finitely many nongeneric points there are at most two valence two or four vertices at the same level or there is a single valence 6 vertex.

We can now prove our first main result.

Proof of Theorem 1.1 Consider the family of sweepouts $\left\{g_{r} \mid r \in[0,1]\right\}$ described in Lemma 5.2. As $g_{0}$ spans $f$ positively and $g_{1}$ spans $f$ negatively there must be some $r$ such that either $g_{r}$ splits $f, g_{r}$ spans $f$ both positively and negatively, or the hypotheses of Lemma 4.3 are satisfied. This is illustrated in Figure 3.

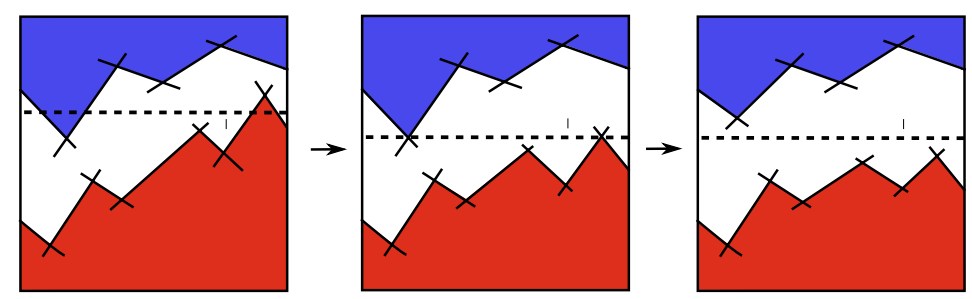

Figure 3

Case 1 The sweepout $g_{r}$ splits $f$. In this case by Theorem 4.2 it follows that $d(\Sigma, T) \leq 2-\chi\left(\Sigma^{\prime}\right)$ so $\chi\left(\Sigma^{\prime}\right) \leq 2-d(\Sigma, T)$.

Case 2 The sweepout $g_{r}$ spans $f$ both positively and negatively. In this case by Theorem 3.1 it follows that $\chi\left(\Sigma^{\prime}\right) \leq 2 \chi(\Sigma)$.

Case 3 There are at most two valence two or four vertices at the same level or there is a valence 6 vertex. By an argument identical to the one in the proof of [5, Lemma 33] it follows that either we are in one of cases 1 or 2 or there is a vertex of valence 4 or valence 6 corresponding to coordinates $(s, t)$ such that for a very small $\epsilon$ the surface $f_{t+\epsilon}^{-1}$ is mostly above $g^{-1}(s)$ and $f_{t-\epsilon}^{-1}$ is mostly below $g^{-1}(s)$. Lemma 4.3 shows that in this case $\chi(\Sigma) \geq-3$ contradicting the hypothesis. 


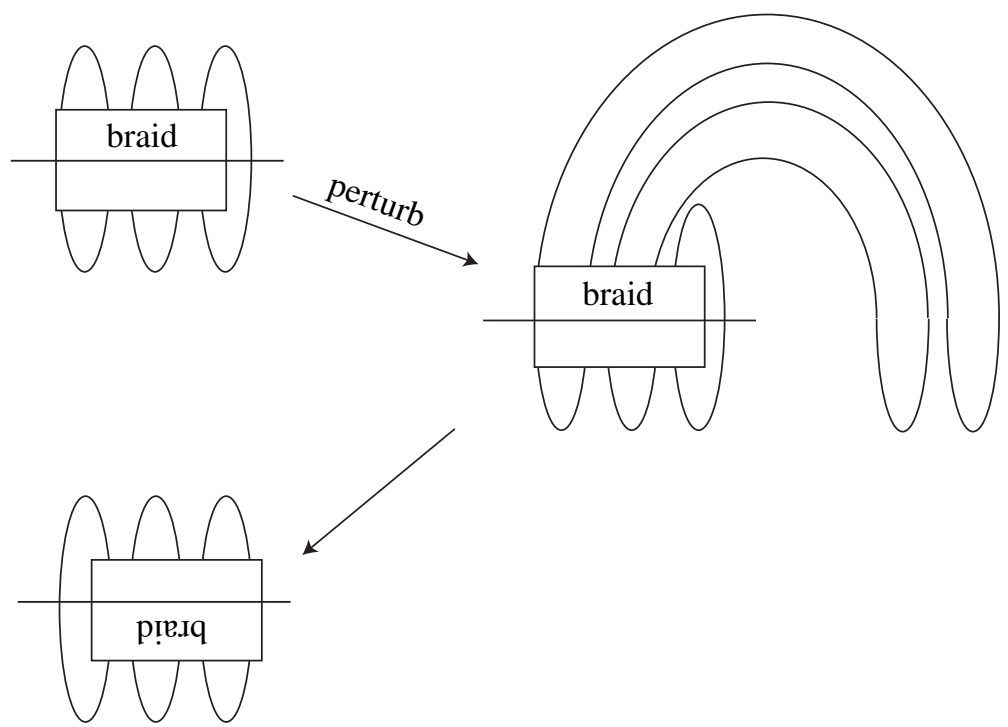

Figure 4

Proof of Corollary 1.2 Figure 4 shows that if $T$ has $n$ bridges with respect to a bridge sphere $\Sigma$, then there is a flippable bridge sphere $\Sigma^{\prime}$ obtained from $\Sigma$ by perturbations with respect to which $T$ has $2 n-1$ bridges. The fact that there is no such bridge sphere with fewer punctures follows by Theorem 1.1.

\section{Bridge surfaces that require a large number of stabiliza- tion and perturbations to become equivalent}

Proof of Theorem 1.3 The proof of this theorem consists of a construction for a pair $(M, K)$ where $K$ is a knot with two distinct bridge surfaces with Euler characteristics $2 s$ and $2 s-2$ respectively so that every common stabilization/perturbation of the two surfaces has Euler characteristic of at most $3 s+4$. In particular this construction gives examples of knots in $S^{3}$ with distinct bridge spheres with bridge number $2 n$ and $2 n-1$, respectively, for which any common perturbation has at least $3 n-4$ bridges.

Let $K$ be a knot in a manifold $M$ and let $\left(\Sigma,\left(H^{+}, \tau^{+}\right),\left(H^{-}, \tau^{-}\right)\right)$be a bridge splitting for $(M, K)$ so that, $\chi(\Sigma) \leq-4$ and $d(\Sigma, K) \geq-3 \chi(\Sigma)$. Such knots can be obtained for example by modifying the construction used in [2] to generate high distance Heegaard splittings by iterating a 2 -fold Dehn twist operator. 
Suppose $f$ is a sweepout for $M$ associated to $\Sigma$. Let $\Gamma_{+}$be a spine of $\left(H^{+}, \tau^{+}\right)$and let $\Gamma_{-}$be a spine of $\left(H^{-}, \tau^{-}\right)$so that $\Gamma_{-}=f^{-1}(-1)$ and $\Gamma_{+}=f^{-1}(1)$. Choose an edge of $\Gamma_{-}$that has a valence 1 vertex, ie, an edge that has one endpoint in $K$. Let $B$ be a ball that is a regular neighborhood of this edge and let $M^{-}$be the closure of $M \backslash B$ containing the one-strand tangle $K^{-}=K \cap M^{-}$. The sweepout $f$ on $M$ can be modified to be a sweepout of $M^{-}$by perturbing $f$ to be constant in $B$. We will use $f$ to refer to either sweepout when the manifold is clear from context.

We will need a new invariant of a surface in $M^{-}$which is very similar to Euler characteristic but does not take into account certain punctures. Consider the manifold $\mathrm{M}^{-}$ described above with spines $\Gamma_{-}$and $\Gamma_{+}$. Then in the complement of $\Gamma_{-} \cup \Gamma_{+}$there are $2 n$ arcs of the knot where $n$ is the bridge number of $K$ in $M$. Of those, $2 n-2$ have one endpoint on $\Gamma_{-}$and one endpoint on $\Gamma_{+}$and 2 arcs have one endpoint on $\Gamma_{+}$and their other endpoint is in the spherical boundary component of $M^{-}$. Let $\kappa_{1}, \ldots, \kappa_{2 n-2}$ be the set of arcs that have both of their endpoints in $\Gamma_{-} \cup \Gamma_{+}$. For any surface $F$ in $M^{-}$, let $\alpha(F)=2 g(F)+\left|F \cap\left(\kappa_{1} \cup \cdots \cup \kappa_{2 n-2}\right)\right|$. This invariant is well defined only up to isotopies of $F$ that are disjoint from $\Gamma_{-} \cup \Gamma_{+}$. Note that $\alpha(F)$ is nonincreasing under c-compressions.

Let $P$ be a manifold homeomorphic to $S^{2} \times I$ containing two vertical $\operatorname{arcs} \tau_{1}$ and $\tau_{2}$. Construct a new manifold $M$ \# $M$ by gluing a copy of $M^{-}$to each of the boundary spheres of $P$ so that the endpoints of each copy of $K^{-}$are identified with one endpoint of $\tau_{1}$ and one endpoint of $\tau_{2}$ to obtain a new knot $K \# K$. Then $(M \# M, K \# K)$ is the connect sum of two copies of $(M, K)$.

We will let $M_{1}^{-}$and $M_{2}^{-}$be the two copies of $M^{-}$in the above construction. We will let $\Sigma_{1}$ and $\Sigma_{2}$ be the copies of $\Sigma$ contained in $M_{1}^{-}$and $M_{2}^{-}$respectively. Finally we will let $f_{1}$ and $f_{2}$ be the sweepouts of $M_{1}^{-}$and $M_{2}^{-}$respectively associated with $\Sigma_{1}$ and $\Sigma_{2}$.

The pair ( $M \# M, K \# K$ ) has two natural generalized Heegaard splittings $\mathcal{H}_{a}$ and $\mathcal{H}_{b}$ induced by the bridge splittings for $M_{1}^{-}, M_{2}^{-}$and $P$, shown in Figures 5 and 6. In both cases we will take $\Sigma_{1}$ and $\Sigma_{2}$ to be the bridge surfaces for $M_{1}^{-}$and $M_{2}^{-}$. However in the first generalized Heegaard splitting we will take the surface $S^{2} \times\{1 / 2\}$ to be the bridge surface for $\left(P, \tau_{1} \cup \tau_{2}\right)$ and for the second one we will take the bridge surface for $P$ to be the surface obtained by tubing together the two spheres which are boundaries of small collars of $S^{2} \times\{0\}$ and $S^{2} \times\{1\}$ respectively along a vertical tube; see Figure 5. Let $\Sigma_{1} \uparrow \uparrow \Sigma_{2}$ and $\Sigma_{1} \uparrow \downarrow \Sigma_{2}$ be the two bridge surfaces for $(M \# M, K \# K)$ obtained by amalgamating $\mathcal{H}_{a}$ and $\mathcal{H}_{b}$ respectively. Note that $\chi\left(\Sigma_{1} \uparrow \uparrow \Sigma_{2}\right)=\chi\left(\Sigma_{1}\right)+\chi\left(\Sigma_{2}\right)=2 \chi(\Sigma)$ and $\chi\left(\Sigma_{1} \uparrow \downarrow \Sigma_{2}\right)=\chi\left(\Sigma_{1}\right)+\chi\left(\Sigma_{2}\right)-2=$ $2 \chi(\Sigma)-2$. 


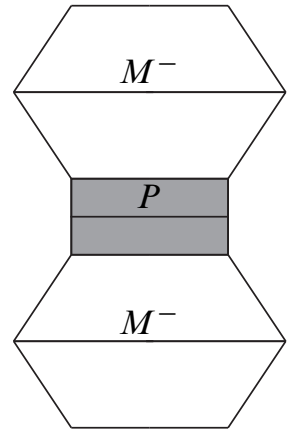

$\mathcal{H}_{1}$

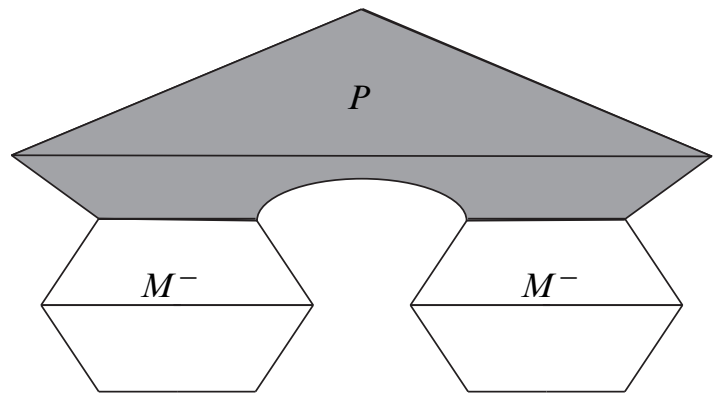

$\mathcal{H}_{2}$

Figure 5: Schematic depiction of $\Sigma_{1} \uparrow \uparrow \Sigma_{2}$ and $\Sigma_{1} \uparrow \downarrow \Sigma_{2}$
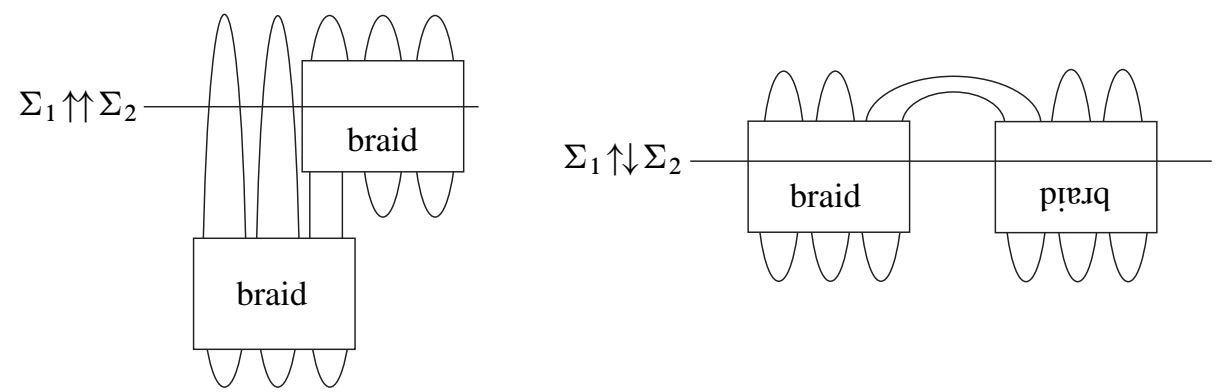

Figure 6: The figure depicts $\Sigma_{1} \uparrow \uparrow \Sigma_{2}$ and $\Sigma_{1} \uparrow \downarrow \Sigma_{2}$ if $M=S^{3}$ and $\Sigma=S^{2}$.

Lemma 6.1 If $\Sigma^{\prime}$ is isotopic to a surface obtained via a sequence of stabilizations and perturbations of $\Sigma_{1} \uparrow \uparrow \Sigma_{2}$ and also to a surface obtained via sequence of stabilizations and perturbations of $\Sigma_{1} \uparrow \downarrow \Sigma_{2}$, then $\chi\left(\Sigma^{\prime}\right) \leq 3 \chi(\Sigma)+4$.

Proof First observe the following:

Remark 6.2 (See [5, Lemma 19].) Let $\left(f_{1} \uparrow \uparrow f_{2}\right)$ be the sweepout associated to $\Sigma_{1} \uparrow \uparrow \Sigma_{2}$ and $\left(f_{1} \uparrow \downarrow f_{2}\right)$ be the sweepout associated to $\Sigma_{1} \uparrow \downarrow \Sigma_{2}$. Then $\left(f_{1} \uparrow \uparrow f_{2}\right)$ spans $f_{1}$ and $f_{2}$ positively and $\left(f_{1} \uparrow \downarrow f_{2}\right)$ spans $f_{1}$ positively and $f_{2}$ negatively.

The remark is clear in the case when $M=S^{3}$ and $\Sigma=S^{2}$, as shown in Figure 6. In the general case the proof is very similar to the proof of [5, Lemma 19] so we leave the details to the reader.

Let $g$ and $g^{\prime}$ be sweepouts for $(M \# M, K \# K)$ defined by perturbing and stabilizing sweepouts $\left(f_{1} \uparrow \uparrow f_{2}\right)$ and $\left(f_{1} \uparrow \downarrow f_{2}\right)$ enough times so that $g$ and $g^{\prime}$ represent isotopic 
bridge decompositions. By Lemma 5.1 it follows that $g$ spans $\left(f_{1} \uparrow \uparrow f_{2}\right)$ positively. By Remark 6.2 it follows that $\left(f_{1} \uparrow \uparrow f_{2}\right)$ spans both $f_{1}$ and $f_{2}$ positively. Therefore we conclude that $g$ spans both $f_{1}$ and $f_{2}$ positively. Similarly, $g^{\prime}$ spans $f_{1}$ positively and $f_{2}$ negatively.

As $g$ and $g^{\prime}$ represent isotopic bridge decompositions, the sweepout $g$ is isotopic to either $g^{\prime}$ or $-g^{\prime}$. In other words, there is a family of sweepouts $\left\{g_{r} \mid r \in[0,1]\right\}$ such that $g_{0}=g, g_{1}= \pm g^{\prime}$. Consider the family of sweepouts $\left\{g_{r} \mid r \in[0,1]\right\}$ described in Lemma 5.2. Because $g^{\prime}$ spans $f_{1}$ positively and $f_{2}$ negatively, the sweepout $g_{1}= \pm g^{\prime}$ spans one of $f_{1}$ or $f_{2}$ positively and the other negatively. Without loss of generality, assume $g_{1}$ spans $f_{1}$ negatively. As $g_{0}$ spans $f_{1}$ positively and $g_{1}$ spans $f_{1}$ negatively, Lemma 5.2 implies that there is an $r$ satisfying one of the following:

Case 1 The sweepout $g_{r}$ splits $f_{1}$ or $g_{r}$ splits $f_{2}$. The argument is the same so suppose $g_{r}$ splits $f_{1}$. In this case by Theorem 4.2 it follows that $d(\Sigma, K) \leq 2-\chi\left(\Sigma^{\prime}\right)$. As $d(\Sigma, K) \geq-3 \chi(\Sigma)$ by construction, it follows that $\chi\left(\Sigma^{\prime}\right) \leq 3 \chi(\Sigma)+4$ as required.

Case 2 The sweepout $g_{r}$ spans $f_{1}$ both positively and negatively and $g_{r}$ spans $f_{2}$, say positively.

By the definition of spanning there exist $s$ and $t_{+}>t_{0}>t_{-}$such that $\left(\Sigma_{1}\right)_{t_{+}}$and $\left(\Sigma_{1}\right)_{t_{-}}$are mostly above $\Sigma_{s}^{\prime} \cap M_{1}^{-}$and $\left(\Sigma_{1}\right)_{t_{0}}$ is mostly below $\Sigma_{s}^{\prime} \cap M_{1}^{-}$and there exist $u$ and $t_{0}^{\prime}<t_{+}^{\prime}$ so that $\left(\Sigma_{2}\right)_{t_{0}^{\prime}}$ is mostly below $\Sigma_{u}^{\prime} \cap M_{2}^{-}$and $\left(\Sigma_{2}\right)_{t_{+}^{\prime}}$ is mostly above $\Sigma_{u}^{\prime} \cap M_{2}^{-}$. As in the proof of Theorem 3.1, there is then a sequence of compressions and cut-compressions of $\Sigma_{s}^{\prime} \cap M_{1}^{-}$in $M_{1}^{-}$after which there are two components of the c-compressed surface $\widetilde{\Sigma_{s}^{\prime}}$ that are parallel to $\Sigma_{1}$ in the complement of the spines. As c-compressions do not increase $\alpha\left(\widetilde{\Sigma_{s}^{\prime}}\right)$ it follows that $2 \alpha(\Sigma) \leq$ $\alpha\left(\widetilde{\Sigma_{s}^{\prime}}\right) \leq \alpha\left(\Sigma_{s}^{\prime} \cap M_{1}^{-}\right)$. Similarly we can conclude that $\alpha(\Sigma) \leq \alpha\left(\widetilde{\Sigma_{u}^{\prime}}\right) \leq \alpha\left(\Sigma_{u}^{\prime} \cap M_{2}^{-}\right)$ where $\widetilde{\Sigma_{u}^{\prime}}$ is the result of c-compressing $\Sigma_{u}^{\prime} \cap M_{2}^{-}$as in Theorem 3.1.

Let $S$ be the decomposing sphere for $(M \# M, K \# K)$ (we may take $S=S^{2} \times\{1 / 2\}$ in $P$ ) and let $N$ be the product region cobounded by $\Sigma_{u}^{\prime}$ and $\Sigma_{s}^{\prime}$. Then $S \cap N$ is a planar surface with at most 2 punctures properly embedded in $N$ which separates $N$ into $N_{1}=N \cap M_{1}^{-}$and $N_{2}=N \cap M_{2}^{-}$. By [9, Lemma 20], it follows that

$$
g\left(M_{1}^{-} \cap \Sigma_{s}^{\prime}\right)+g\left(M_{2}^{-} \cap \Sigma_{u}^{\prime}\right) \leq g\left(\Sigma^{\prime}\right) .
$$

Let $\Gamma_{+}^{\prime}$ and $\Gamma_{-}^{\prime}$ be the spines for $\Sigma^{\prime}$. If some strand of $K_{1} \# K_{2}-\left(\Gamma_{+}^{\prime} \cup \Gamma_{-}^{\prime}\right)$ intersects both $\Sigma_{s}^{\prime} \cap M_{1}$ and $\Sigma_{u}^{\prime} \cap M_{2}$ then this strand must also intersect the decomposing sphere. As the decomposing sphere has only two punctures it follows that

$$
\left|\Sigma^{\prime} \cap\left(K_{1} \# K_{2}\right)\right|=\left|\Sigma_{s}^{\prime} \cap M_{1} \cap\left(K_{1} \# K_{2}\right)\right|+\left|\Sigma_{u}^{\prime} \cap M_{2} \cap\left(K_{1} \# K_{2}\right)\right|-2 .
$$


Let $\kappa$ and $\kappa^{\prime}$ be the two arcs of $K_{1} \# K_{2}$ in the complement of the spines of $\Sigma_{1}$ and $\Sigma_{2}$ that intersect $S$. From Equation (1) and Equation (2) it follows that

$$
\begin{aligned}
& 2 g\left(\Sigma^{\prime}\right)+\left|\Sigma^{\prime} \cap\left(K_{1} \# K_{2}\right)\right| \\
& \geq 2\left(g\left(M_{1}^{-} \cap \Sigma_{s}^{\prime}\right)+g\left(M_{2}^{-} \cap \Sigma_{u}^{\prime}\right)\right)+\left|\Sigma_{s}^{\prime} \cap M_{1} \cap\left(K_{1} \# K_{2}-\left(\kappa \cup \kappa^{\prime}\right)\right)\right| \\
& +\left|\Sigma_{u}^{\prime} \cap M_{2} \cap\left(K_{1} \# K_{2}-\left(\kappa \cup \kappa^{\prime}\right)\right)\right|-2 \\
& =\alpha\left(M_{1}^{-} \cap \Sigma_{s}^{\prime}\right)+\alpha\left(M_{2}^{-} \cap \Sigma_{u}^{\prime}\right)-2 .
\end{aligned}
$$

Using the discussion in the first paragraph of this case, it follows that

$$
\begin{aligned}
\alpha\left(M_{1}^{-} \cap \Sigma_{s}^{\prime}\right)+\alpha\left(M_{2}^{-} \cap \Sigma_{u}^{\prime}\right)-2 & \geq \alpha\left(\widetilde{\Sigma_{s}^{\prime}}\right)+\alpha\left(\widetilde{\Sigma_{u}^{\prime}}\right)-2 \\
& \geq 2 \alpha\left(\Sigma_{1}\right)+\alpha\left(\Sigma_{2}\right)-2
\end{aligned}
$$

Recall that $\Sigma_{1}$ and $\Sigma_{2}$ are copies of the same surface $\Sigma$ which has $2 n$ punctures. Of those, two are not counted in the computation of $\alpha$ so it follows that

$$
2 \alpha\left(\Sigma_{1}\right)+\alpha\left(\Sigma_{2}\right)-2=3(\alpha(\Sigma))-2=6 g(\Sigma)+6 n-8 .
$$

We conclude that

$$
2 g\left(\Sigma^{\prime}\right)+\left|\Sigma^{\prime} \cap\left(K_{1} \# K_{2}\right)\right| \geq 6 g(\Sigma)+6 n-8 .
$$

It therefore follows that, as desired,

$$
\chi\left(\Sigma^{\prime}\right)=2-2 g\left(\Sigma^{\prime}\right)-\left|\Sigma^{\prime} \cap\left(K_{1} \# K_{2}\right)\right| \leq 2-6 g(\Sigma)-6 n+8
$$

$$
=3(2-2 g(\Sigma)-2 n))+4=3 \chi(\Sigma)+4 \text {. }
$$

Case 3 There are at most two valence two or valence four vertices at the same level or there is a valence 6 vertex. As in Theorem 1.1 this implies that $\chi(\Sigma) \geq-3$ contrary to our hypothesis.

Theorem 1.3 suggests that the bound obtained above can be improved as follows:

Conjecture 6.3 There exist infinitely many manifolds $M_{\alpha}$ each containing a knot $K_{\alpha}$ so that each pair $\left(M_{\alpha}, K_{\alpha}\right)$ has two bridge surfaces $\Sigma$ and $\Sigma^{\prime}$ with $\chi(\Sigma)=2 s$ and $\chi\left(\Sigma^{\prime}\right)=2 s-2$ so that if $\Sigma^{\prime \prime}$ is a bridge surface that is isotopic to stabilizations and perturbations of both $\Sigma$ and $\Sigma^{\prime}$, then $\chi\left(\Sigma^{\prime \prime}\right) \leq 3 s$.

The main obstacle to proving this conjecture is Case 2 of Lemma 6.1. As cutcompressing can in fact increase the number of punctures of a surface current techniques are insufficient to further sharpen this bound.

Acknowledgement During the preparation of this manuscript both authors were supported by NSF grant numbers DMS0704207 and DMS0602638. 


\section{References}

[1] D Bachman, Heegaard splittings of sufficiently complicated 3-manifolds I: Stabilization arXiv:0903.1695

[2] T Evans, High distance Heegaard splittings of 3-manifolds, Topology Appl. 153 (2006) 2631-2647 MR2243739

[3] J Hass, A Thompson, W Thurston, Stabilization of Heegaard splittings, Geom. Topol. 13 (2009) 2029-2050 MR2507114

[4] C Hayashi, K Shimokawa, Heegaard splittings of trivial arcs in compression bodies, J. Knot Theory Ramifications 10 (2001) 71-87 MR1822141

[5] J Johnson, Bounding the stable genera of Heegaard splittings from below, J. Topol. 3 (2010) 668-690 MR2684516

[6] K Reidemeister, Zur dreidimensionalen Topologie, Abh. Math. Sem. Univ. Hamburg 11 (1933) 189-194

[7] M Scharlemann, M Tomova, Uniqueness of bridge surfaces for 2-bridge knots, Math. Proc. Cambridge Philos. Soc. 144 (2008) 639-650 MR2418708

[8] J Singer, Three-dimensional manifolds and their Heegaard diagrams, Trans. Amer. Math. Soc. 35 (1933) 88-111 MR1501673

[9] K Takao, A refinement of Johnson's bounding for the stable genera of Heegaard splittings, Osaka J. Math. 48 (2011) 251-268

[10] M Tomova, Multiple bridge surfaces restrict knot distance, Algebr. Geom. Topol. 7 (2007) 957-1006 MR2336246

[11] M Tomova, Thin position for knots in a 3-manifold, J. Lond. Math. Soc. (2) 80 (2009) 85-98 MR2520379

Mathematics Department, Oklahoma State University 401 Mathematical Sciences, Stillwater OK 74078-1058, USA

Department of Mathematics, The University of Iowa Iowa City IA 52242, USA

jjohnson@math.okstate.edu, mtomova@math.uiowa.edu http://www.math.okstate.edu/ jjohnson/, http://www . math . uiowa. edu/ mtomova

Received: 17 April $2010 \quad$ Revised: 29 March 2011 